\title{
High harmonic generation on noble gas clusters
}

\author{
BalázS Bódi, ${ }^{1}$ MÁRK Aladi, ${ }^{1}$ Péter RÁCZ, ${ }^{1}$ ISTVÁN B.

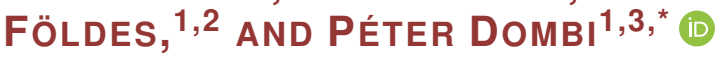 \\ ${ }^{1}$ Wigner Research Centre for Physics, H-1121 Budapest, Hungary \\ ${ }^{2}$ University of Szeged, H-6720 Szeged, Hungary \\ ${ }^{3}$ ELI-ALPS Research Institute, H-6728 Szeged, Hungary \\ *dombi.peter@wigner.mta.hu
}

\begin{abstract}
High order harmonics (HHG) were generated on noble gas cluster targets with different cluster sizes. The independently characterized cluster sources enabled experimental investigation of the recombination mechanism. HHG spectra were recorded for different backing pressures and gases ( $\mathrm{Ar}, \mathrm{Xe}$ ) as a function of driver pulse ellipticity. Since the ellipticity-dependent HHG decay is essentially the same for the different gas-pressure pairs, we can conclude that the recombination process is dominated by atom-to-itself recollisions irrespective of cluster size and material.
\end{abstract}

(C) 2019 Optical Society of America under the terms of the OSA Open Access Publishing Agreement

\section{Introduction}

High-order harmonic generation (HHG) has emerged as a tool for creating remarkably short extreme ultraviolet (XUV) pulses [1]. The interaction of atomic gases and intense ultrashort laser pulses leading to HHG and attosecond pulse generation was first understood as a semi-classical three-step process involving tunnel ionization, free-space electron motion and recombination [2-4]. HHG proved to be an efficient tool for the generation of attosecond pulses [5-8] (with the help of carrier-envelope phase-stabilized, few-cycle-pulse laser technology $[9,10])$ and by proper filtering of the XUV spectrum, single pulses with less than 100 asec duration are achievable [11]. The recolliding electron can also enable molecular tomography [12] and other ultrafast science applications [1]. Further development of HHG sources recently involved infrared drivers [13], loose focusing of high-power beams [14], optimized quasi phase-matching [15] and using synthesized waveforms as drivers $[16,17]$.

It follows from the simplified semi-classical picture that practically only a linearly polarized driver is suitable for HHG, because otherwise the electron cannot hit the parent ion. In a more proper quantum mechanical picture, the extension of the wavefunction of the returning electron results in HHG signal decay as a function of the driver pulse ellipticity $[18,19]$ with the recollision process being less likely for increasing ellipticity. Recent experiments defined the threshold ellipticity $\left(\varepsilon_{\mathrm{th}}\right)$ as the half-FWHM of this decay [20-23].

The use of clusters as HHG targets has a lot of potential compared to atomic monomers, e.g clusters provide higher photon yield [24-26] and higher cutoff [26,27], although there is also ambiguity in the literature concerning the latter [28,29]. Some numerical studies [30] predicted an extended cutoff even beyond a photon energy corresponding to $I_{\mathrm{p}}+8 U_{\mathrm{p}}$, but this was never confirmed by experiments [26,27]. (Here, $I_{\mathrm{p}}$ indicates the ionization potential and $U_{\mathrm{p}}$ is the ponderomotive energy). However, a cutoff well above the usual $I_{\mathrm{p}}+3.17 U_{\mathrm{p}}$ was demonstrated in [25]. Additionally, for clusters, the HHG intensity shows a steeper dependence with the laser intensity than for the case of atoms [31] which was also seen in our previous experiments [25]. It is also known that the degree of linear polarization of the XUV light is decreased if cluster targets are used [23]. In summary, the differences of cluster HHG are rooted in the almost solid density of clusters which can result in efficient (>90\%) absorption of laser light [32], and thus higher conversion efficiency. 
However, a fundamental research question related to cluster-based HHG remained, namely whether the electron recombines to the parent ion in a cluster ("atom-to-itself" recombination) or recollision with neighbouring atoms is also possible resulting in "atom-to-neighbour recombination". This possible scenario was indirectly studied before [28,29] and further support for this hypothesis was provided by independent theory work [33-36]. It is also possible that harmonics come from a delocalized wavefunction over the whole cluster and to which they recombine coherently after tunneling out ("cluster-to-itself" recombination) [23]. For the latter two mechanisms, one would expect the increase of the threshold ellipticity.

Here, we perform HHG experiments on various cluster targets to determine the mechanism of recombination and establish whether the dominant recombination scenario is cluster-to-itself, atom-to-neighbour or atom-to-itself.

\section{Experimental setup}

An amplified Ti:sapphire laser was used for the experiments providing 2.5-mJ pulses with 1 $\mathrm{kHz}$ repetition rate. A $\lambda / 4$ plate is inserted into this beam to enable a continuous transition of the HHG driver pulse from linear polarization toward circular polarization. At the entrance of the target chamber the pulses were $78 \pm 2$ fs long (intensity FWHM), with a beam diameter of $1 \mathrm{~cm}$ and we attenuated them to $1.25 \mathrm{~mJ}$ pulse energy. This beam was focused into a vacuum chamber $\left(\mathrm{p}<\sim 10^{-2} \mathrm{~Pa}\right.$ ) using an anti-reflection coated lens with $30 \mathrm{~cm}$ focal length (Fig. 1). The laser axis was set to be $1 \mathrm{~mm}$ from the nozzle exit and the focal spot was $6 \pm 0.5 \mathrm{~mm}$ before the jet, resulting in an intensity of $(1.42 \pm 0.22) \times 10^{14} \mathrm{~W} / \mathrm{cm}^{2}$ that was kept constant during the measurements. Thus, the applied intensity was chosen to be near to the optimal conversion rate [25], which is more than a factor 2 higher than the intensity for which the effect of nanoplasma inside the cluster was demonstrated [37,38] This arrangement of the jet and focus selects short trajectories against long ones [39].

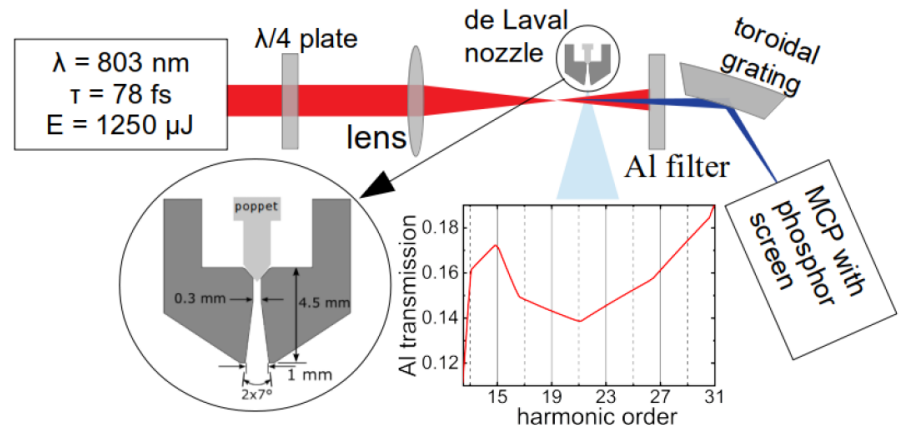

Fig. 1. The experimental setup. We focused an ultrashort IR pulse close to the nozzle exit, and filtered with $0.8 \mu \mathrm{m} \mathrm{Al}$, measuring the resulting XUV signal with an MCP detector. See inset for jet details and $\mathrm{Al}$ transmission profile showing the used wavelength interval.

Gas clusters were injected into the chamber with a commercial valve (Parker series 9) using a supersonic de Laval nozzle which is designed for laser-plasma experiments [40]. This scheme provided gas jets with density fluctuations below the 5\% level, see Fig. 1(inset). We used Ar and Xe gas at backing pressures of 1, 10, 20 bars (Ar) and 1, 5, 10, 14 bars (Xe), respectively. The cluster sizes were determined by Rayleigh scattering diagnostics [38]. Assuming spherical clusters, an approximate diameter can be calculated with the van der Waals radii [41]: $1.88 \AA$ for Ar and $2.16 \AA$ for Xe (see Fig. 2 for details). The generated XUV light was filtered with a $0.8 \mu \mathrm{m}$ thick Al foil to discard the fundamental wavelength. The XUV signal was characterized with a toroidal holographic grating (Jobin Yvon, 550 lines $/ \mathrm{mm}$ ), and the spectrum was recorded with a microchannel plate (MCP), phosphor screen and CCD camera. 


\section{Optics EXPRESS}
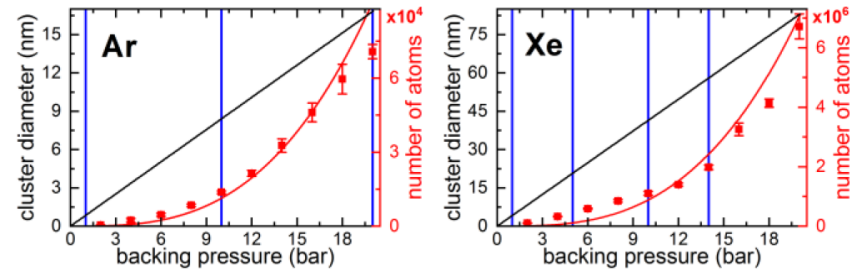

Fig. 2. Cluster dimensions. The scatter plots show the measurements of cluster sizes as a function of the backing pressure for the nozzle used. The number of atoms within the cluster (red squares) were fit (red lines) and converted to cluster diameter (black lines). The pressure values used in this study are marked with vertical blue lines.

\section{Results and discussion}

The experiment was performed by measuring HHG spectra as a function of the driver pulse ellipticity that was changed in small steps using the $\lambda / 4$ plate. Figure 3 shows averaged spectra as a function of ellipticity for different gases (Xe and Ar) and pressures.

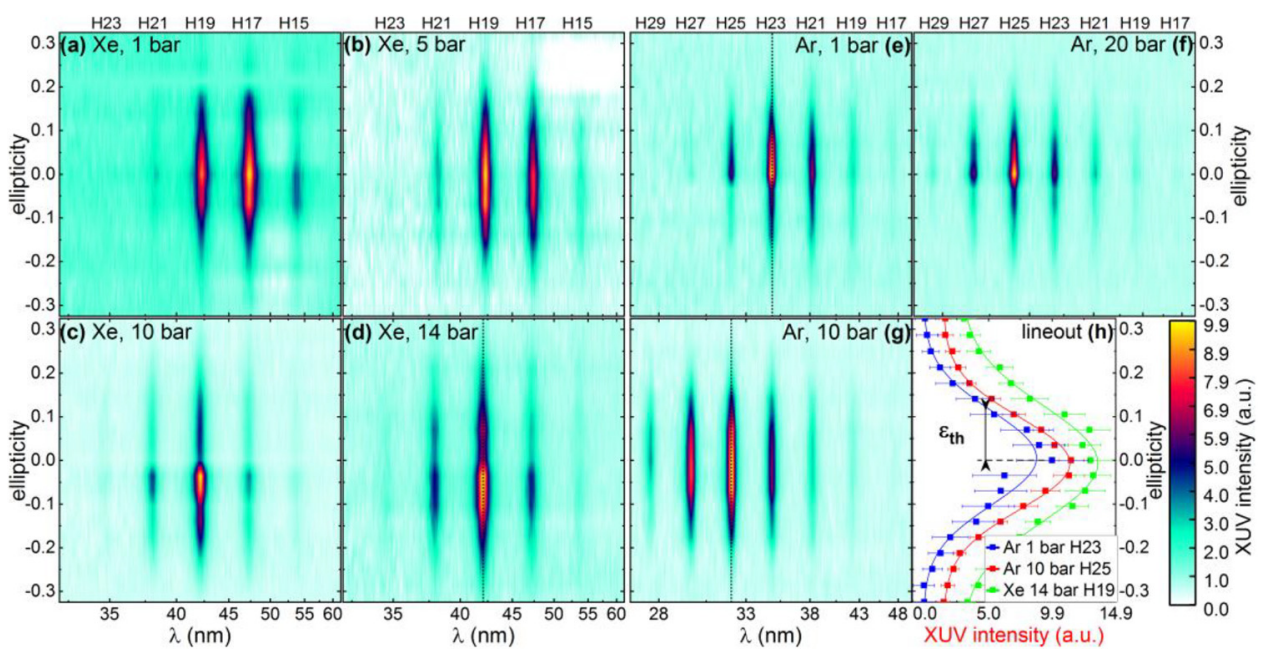

Fig. 3. HHG spectra as a function of the driver pulse ellipticity for different gases and backing pressures. Xe with (a) 1 bar, (b) 5 bar, (c) 10 bar and (d) 14 bar backing pressures; and Ar with (e) 1 bar, (g) 10 bar and (f) 20 bar backing pressures. The lineouts for Ar 1and 10 bar and Xe 14 bar are presented in (h), showing the evaluation method of threshold ellipticity, $\varepsilon_{\text {th }}$ to be used in Fig. 4. Threshold ellipticities in (h) are $0.12,0.12$ and 0.14 , respectively. For better visibility, curves in (h) are offset by 1.5 units each.

Whereas in Xe the cutoff frequency shows an increase with increasing backing pressure, this is not so clear in high-pressure Ar, as it shows saturation. A possible explanation is that the conditions for ideal phase matching may improve with increasing backing pressure, similarly to atoms [29]. Additionally, the monotonic increase for the largest clusters can partly be caused by increasing space charge of multiply ionized Xe (providing the largest clusters), in accordance with results of Ruf et al. on multiply ionized $\mathrm{Kr}$ clusters [23].

The cutoff law of $\mathrm{I}_{p}+3.17 \mathrm{U}_{p}$ predicts $42.6 \mathrm{eV}$ for $\mathrm{Ar}$ and $39.0 \mathrm{eV}$ for Xe. With increasing backing pressure, the conditions for ideal phase-matching are approached. Each dataset is an average of between 20 and 28 shots. We fitted Gaussians for each harmonic order and backing pressure and thus we gained threshold ellipticity values $\varepsilon_{\text {th }}$ [20], see Fig. 3(h). Figure 4 shows 
$\varepsilon_{\text {th }}$ as a function of harmonic order for each nozzle setting. The decreasing signal-noise ratio towards the sides of the spectra (H15 and H23 for xenon, H17 and H29 for argon) indicates larger error bars compared to the ones in the centre with strongest signal.

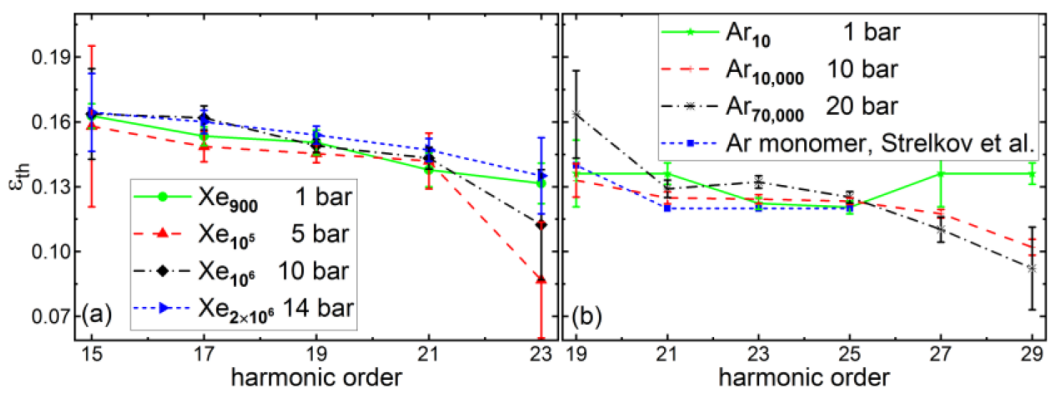

Fig. 4. The ellipticity threshold parameter for all studied gases and pressures. (a) Xe H15-H23, (b) Ar H19-H29. Reference values for Ar monomers are taken from Ref. [20] and plotted as a blue dashed curve in (b).

Figure 4 shows that $\varepsilon_{\text {th }}$ depends practically neither on the backing pressure nor the gas used (Xe/Ar). Threshold ellipticity values match within the uncertainty level of our measurement for both cases and for all pressures used during our experiments. We also found that our results for the threshold ellipticity are fully in line with similar independent measurements using Ar monomers by Strelkov et al. [20], see Fig. 4(b).

Even though the density of clusters is increased together with the pressure, we can conclude, that the pressure independence of the threshold means that the cluster size does not change the ellipticity dependence of the HHG signal. Our datasets cover a huge range in cluster size from $0.8 \mathrm{~nm}$ (Ar, 1 bar, $\sim 11$ atoms) to $58 \mathrm{~nm}$ (Xe, 14 bar, $2.4 \times 10^{6}$ atoms) and they all have a similar ellipticity decay (within error) for all harmonics that we recorded.

Previous results about recombination in clusters [23] suggest three different mechanisms, namely cluster-to-itself, atom-to-neighbour and atom-to-itself recollision and recombination. The first one can be clearly ruled out, since much larger clusters do not give more signal for higher values of the ellipticity. Our measurements are above the single atom regime, with the smallest cluster size having $\sim 11$ atoms (with a certain approximation for smaller clusters, since the Rayleigh scattering method can safely identify clusters above $\sim 100$ atoms [38,42]). Knowing this, we cannot fully exclude atom-to-neighbour recombination mechanisms, however we also have to consider that our results agree very well with those of Strelkov et al. [20] using Ar monomers (atomic targets), as shown in Fig. 4(b). With these aspects considered, we can conclude that the recombination mechanism in cluster HHG is taking place dominantly via the atom-to-itself channel and other contributions are negligible.

This conclusion is further supported by a very simple classical trajectory analysis of photoionized electrons as a function of ionization instant. Electron trajectories are initialized at the outermost edge of clusters of three different sizes $(0.8 \mathrm{~nm}, 4.1 \mathrm{~nm}$ and $8.4 \mathrm{~nm})$ and then propagated in the external laser field having $1.4 \times 10^{14} \mathrm{~W} / \mathrm{cm}^{2}$ peak intensity and 78 fs pulse length (as in our experiments). If the electron is driven back to any position in the cluster, we record the return kinetic energy and examine where the electron hits the cluster again. If the electron returns to a characteristic volume belonging to the parent ion in the cluster (evaluated from data in Fig. 2), we consider this as an atom-to-itself recollision, all other recollision events are considered as cluster-to-itself. Atom-to-neighbour events are not distinguished here. Return energy plots for cluster sizes of $0.8 \mathrm{~nm}, 4.1 \mathrm{~nm}$ and $8.4 \mathrm{~nm}$ and different ellipticities are shown in Visualization 1. Zero return energy is also plotted if the test electron does not return to the cluster. It can be seen that return energy events decrease as the ellipticity increases especially 
for larger clusters, as expected. We also calculated the ratio of return events with respect to all events (that is, returns and events when the electron misses the cluster). We call this quantity "return fraction" which is also plotted in Visualization 1 as a function of ellipticity. (Here, only atom-to-itself and cluster-to-itself events are distinguished.)

The FWHM of these return fraction curves provide a rough first estimate on the threshold ellipticity one can expect. This showed us that if we consider trajectories returning to the parent ion, an $\varepsilon_{\text {th }}$ of $\sim 0,15$ can be expected (black curves in Visualization 1), in line with our experimental observations. Cluster-to-itself recollision and recombination (if present, at all) can extend the threshold ellipticity substantially, even up to $\varepsilon_{\text {th }}=1$ starting at a cluster size of $\sim 8 \mathrm{~nm}$. This range is well covered by our experiments. Since the increase of threshold ellipticity was not observed experimentally, we can rule out the cluster-to-itself mechanism.

This is in line with the intuitive argument of the weak binding forces of the cluster which do not enable significant delocalization during the recollision time. Even for the largest cluster sizes that we tested involving millions of atoms, our results are essentially underpinning the dominance of the atom-to-itself recombination mechanism.

\section{Summary}

In summary, we tested high harmonic generation on Ar and Xe cluster targets experimentally in a wide range of cluster sizes, ranging from $\sim 10$ to several millions of atoms to study the presence of different recombination mechanisms, namely atom-to-itself, atom-to-neighbour and cluster-to-itself. By analyzing the ellipticity dependendence of the HHG signal, we found that during the HHG process on noble gas clusters, the recombination process is dominated by the atom-to-itself recollision mechanism and therefore the laser-induced charge state does not significantly delocalize within the cluster during the recollision time.

\section{Funding}

PETACom project FET Open program (829153); Nemzeti Kutatási Fejlesztési és Innovációs Hivatal (VEKOP-2.3.2-16-2017-00015); Hungarian Scientific Research Fund (FK125270, PD125249, OTKA113222); European Social Fund (EFOP-3.6.2-16-2017-00005).

\section{References}

1. F. Krausz and M. Ivanov, “Attosecond physics," Rev. Mod. Phys. 81(1), 163-234 (2009). and references therein.

2. M. Lewenstein, P. Balcou, M. Yu. Ivanov, A. L'Huillier, and P. B. Corkum, "Theory of high-harmonic generation by low-frequency laser fields," Phys. Rev. A 49(3), 2117-2132 (1994).

3. J. L. Krause, K. J. Schafer, and K. C. Kulander, "High-order harmonic generation from atoms and ions in the high intensity regime," Phys. Rev. Lett. 68(24), 3535-3538 (1992).

4. P. B. Corkum, "Plasma perspective on strong field multiphoton ionization," Phys. Rev. Lett. 71(13), 1994-1997 (1993).

5. G. Farkas and C. Tóth, "Proposal for attosecond light pulse generation using laser induced multiple-harmonic conversion processes in rare gases," Phys. Lett. A 168(5-6), 447-450 (1992).

6. N. A. Papadogiannis, B. Witzel, C. Kalpouzos, and D. Charalambidis, "Observation of Attosecond Light Localization in Higher Order Harmonic Generation," Phys. Rev. Lett. 83(21), 4289-4292 (1999).

7. M. Hentschel, R. Kienberger, C. Spielmann, G. A. Reider, N. Milosevic, T. Brabec, P. Corkum, U. Heinzmann, M. Drescher, and F. Krausz, “Attosecond metrology,” Nature 414(6863), 509-513 (2001).

8. E. Goulielmakis, M. Uiberacker, R. Kienberger, A. Baltuska, V. Yakovlev, A. Scrinzi, T. Westerwalbesloh, U. Kleineberg, U. Heinzmann, M. Drescher, and F. Krausz, "Direct Measurement of Light Waves," Science 305(5688), 1267-1269 (2004).

9. P. Dombi, A. Apolonski, C. Lemell, G. G. Paulus, M. Kakehata, R. Holzwarth, T. Udem, K. Torizuka, J. Burgdorfer, T. W. Hansch, and F. Krausz, "Direct measurement and analysis of the carrier-envelope phase in light pulses approaching the single-cycle regime," New J. Phys. 6, 39 (2004).

10. P. Dombi, V. S. Yakovlev, K. O'Keeffe, T. Fuji, M. Lezius, and G. Tempea, "Pulse compression with time-domain optimized chirped mirrors," Opt. Express 13(26), 10888-10894 (2005). and references therein.

11. K. Zhao, Q. Zhang, M. Chini, Y. Wu, X. Wang, and Z. Chang, "Tailoring a 67 attosecond pulse through advantageous phase-mismatch," Opt. Lett. 37(18), 3891-3893 (2012). 
12. J. Itatani, J. Levesque, D. Zeidler, H. Niikura, H. Pépin, J. C. Kieffer, P. B. Corkum, and D. M. Villeneuve, "Tomographic imaging of molecular orbitals," Nature 432(7019), 867-871 (2004).

13. T. Popmintchev, M.-C. Chen, D. Popmintchev, P. Arpin, S. Brown, S. Ališauskas, G. Andriukaitis, T. Balčiunas, O. D. Mücke, A. Pugzlys, A. Baltuška, B. Shim, S. E. Schrauth, A. Gaeta, C. Hernández-García, L. Plaja, A. Becker, A. Jaron-Becker, M. M. Murnane, and H. C. Kapteyn, "Bright Coherent Ultrahigh Harmonics in the keV X-ray Regime from Mid-Infrared Femtosecond Lasers," Science 336(6086), 1287-1291 (2012).

14. A. Nayak, I. Orfanos, I. Makos, M. Dumergue, S. Kühn, E. Skantzakis, B. Bódi, K. Varjú, C. Kalpouzos, H. I. B. Banks, A. Emmanouilidou, D. Charalambidis, and P. Tzallas, "Multiple ionization of argon via multi-XUV-photon absorption induced by 20-GW high-order harmonic laser pulses," Phys. Rev. A 98(2), 023426 (2018).

15. P. Rudawski, C. M. Heyl, F. Brizuela, J. Schwenke, A. Persson, E. Mansten, R. Rakowski, L. Rading, F. Campi, B. Kim, P. Johnsson, and A. L'Huillier, “A high-flux high-order harmonic source,” Rev. Sci. Instrum. 84(7), 073103 (2013).

16. E. Balogh, B. Bódi, V. Tosa, E. Goulielmakis, K. Varjú, and P. Dombi, "Genetic optimization of attosecond-pulse generation in light-field synthesizers," Phys. Rev. A 90 (2), 023855 (2014).

17. B. Bódi, E. Balogh, V. Tosa, E. Goulielmakis, K. Varjú, and P. Dombi, "Attosecond pulse generation with an optimization loop in a light-field-synthesizer," Opt. Express 24(19), 21957-21962 (2016).

18. N. Hay, R. de Nalda, T. Halfmann, K. J. Mendham, M. B. Mason, M. Castillejo, and J. P. Marangos, "High-order harmonic generation from organic molecules in ultra-short pulses," Eur. Phys. J. D 14(2), 231-240 (2001).

19. A. Flettner, J. König, M. B. Mason, T. Pfeifer, U. Weichmann, and G. Gerber, "Atomic and molecular high-harmonic generation: A comparison of ellipticity dependence based on the three-step model," J. Mod. Opt. 50(3-4), 529-537 (2003).

20. V. V. Strelkov, M. A. Khokhlova, A. A. Gonoskov, I. A. Gonoskov, and M. Yu. Ryabikin, "High-order harmonic generation by atoms in an elliptically polarized laser field: Harmonic polarization properties and laser threshold ellipticity,” Phys. Rev. A 86(1), 013404 (2012).

21. E. W. Larsen, S. Carlström, E. Lorek, C. M. Heyl, D. Paleček, K. J. Schafer, A. L'Huillier, D. Zigmantas, and J. Mauritsson, "Sub-cycle ionization dynamics revealed by trajectory resolved, elliptically-driven high-order harmonic generation," Sci. Rep. 6(1), 39006 (2016).

22. P. M. Abanador, F. Mauger, K. Lopata, M. B. Gaarde, and K. J. Schafer, "Semiclassical modeling of high-order harmonic generation driven by an elliptically polarized laser field: the role of recolliding periodic orbits," J. Phys. B At., Mol. Opt. Phys. 50(3), 035601 (2017).

23. H. Ruf, C. Handschin, R. Cireasa, N. Thiré, A. Ferré, S. Petit, D. Descamps, E. Mével, E. Constant, V. Blanchet, B. Fabre, and Y. Mairesse, "Inhomogeneous High Harmonic Generation in Krypton Clusters," Phys. Rev. Lett. 110(8), 083902 (2013).

24. J. W. G. Tisch, T. Ditmire, D. J. Fraser, N. Hay, M. B. Mason, E. Springate, J. P. Marangos, and M. H. R. Hutchinson, "Investigation of high-harmonic generation from xenon atom clusters," J. Phys. B: At., Mol. Opt. Phys. 30(20), L709-L714 (1997).

25. M. Aladi, I. Márton, P. Rácz, P. Dombi, and I.B. Földes, "High harmonic generation and ionization effects in cluster targets," High Power Laser Sci. Eng. 2, E32 (2014).

26. C. Vozzi, M. Nisoli, J-P. Caumes, G. Sansone, S. Stagira, S. De Silvestri, M. Vecchiocattivi, D. Bassi, M. Pascolini, L. Poletto, P. Villoresi, and G. Tondello, "Cluster effects in high-order harmonics generated by ultrashort light pulses," Appl. Phys. Lett. 86(11), 111121 (2005).

27. L. Feng and H. Liu, "Attosecond extreme ultraviolet generation in cluster by using spatially inhomogeneous field," Phys. Plasmas 22(1), 013107 (2015).

28. H. Park, Z. Wang, H. Xiong, S. B. Schoun, J. Xu, P. Agostini, and L. F. DiMauro, "Size-Dependent High-order Harmonic Generation in Rare-Gas Clusters," Phys. Rev. Lett. 113(26), 263401 (2014).

29. Y. Tao, R. Hagmeijer, H. M. J. Bastiaens, S. J. Goh, P. J. M. van der Slot, S. G. Biedron, S. V. Milton, and K.-J. Boller, "Cluster size dependence of high-order harmonic generation," New J. Phys. 19(8), 083017 (2017).

30. J. R. Vázquez de Aldana and L. Roso, "High-order harmonic generation in atomic clusters with a two-dimensional model," J. Opt. Soc. Am. B 18(3), 325-330 (2001).

31. T. D. Donnelly, T. Ditmire, K. Neumann, M. D. Perry, and R. W. Falcone, "High-Order Harmonic Generation in Atom Clusters," Phys. Rev. Lett. 76(14), 2472-2475 (1996).

32. T. Ditmire, R. A. Smith, J. W. G. Tisch, and M. H. R. Hutchinson, "High Intensity Laser Absorption by Gases of Atomic Clusters," Phys. Rev. Lett. 78(16), 3121-3124 (1997).

33. P. Moreno, L. Plaja, and L. Roso, "High-Order Harmonic Generation by Electron-Proton Recombination,” Europhys. Lett. 28(9), 629-633 (1994).

34. S. X. Hu and Z. Z. Xu, "Dynamics of an intense laser-driven multiwell system: A model of ionized clusters," Phys. Rev. A 56(5), 3916-3922 (1997).

35. V. Véniard, R. Taïeb, and A. Maquet, "Atomic clusters submitted to an intense short laser pulse: A density-functional approach,” Phys. Rev. A 65(1), 013202 (2001).

36. D. F. Zaretsky, P. Korneev, and W. Becker, "High-order harmonic generation in clusters irradiated by an infrared laser field of moderate intensity," J. Phys. B: At., Mol. Opt. Phys. 43(10), 105402 (2010).

37. M. Aladi, R. Bolla, P. Rácz, and I. B. Földes, "Noble gas clusters and nanoplasmas in high harmonic generation," Nucl. Instrum. Methods Phys. Res., Sect. B 369, 68-71 (2016). 


\section{Optics EXPRESS}

38. M. Aladi, R. Bolla, D. Cardenas, L. Veisz, and I.B. Földes, "Cluster size distribution in gas jets for different nozzle geometries," J. Instrum. 12(06), C06020 (2017).

39. M. Bellini, C. Lyngå, A. Tozzi, M. B. Gaarde, T. W. Hänsch, A. L'Huillier, and C.-G. Wahlström, "Temporal Coherence of Ultrashort High-Order Harmonic Pulses," Phys. Rev. Lett. 81(2), 297-300 (1998).

40. K. Schmid and L. Veisz, "Supersonic gas jets for laser-plasma experiments," Rev. Sci. Instrum. 83(5), 053304 (2012).

41. A. Bondi, "Van der Waals volumes and radii," J. Phys. Chem. 68(3), 441-451 (1964).

42. Zhiyuan Chen, Dong Liu, Jifeng Han, and Lixin Bai, "The optical measurement of large cluster tracks in a gas jet," Sci. Rep. 6(1) 32391 (2016) 